\title{
Análisis de la necesidad de la educación financiera en la formación colegial ${ }^{1}$
}

\section{Analysis of the need for financial education in collegiate formation}

\author{
Jairo Beymar Céspedes López ${ }^{2}$
}

\begin{abstract}
RESUMEN
La educación financiera es un factor importante en la vida de cualquier persona, el consumo, el ahorro, la inversión y otros conceptos básicos de las finanzas son desconocidos por gran parte de la población en el mundo, el presente artículo identifica ciertas actitudes frente a ello y propone de manera básica pilares fundamentales de la educación financiera que podrían ser implementados en la educación secundaria, considerando que esta debería ser implementada como materia curricular en la educación.
\end{abstract}

Palabras clave: Educación financiera; administración de recursos; finanzas. Clasificación JEL: G00, I21, I22.

1 El artículo se origina a través de la percepción de la necesidad de educación y cultura financiera tanto en jóvenes como en adultos no solamente de Bolivia sino también de distintos lugares de Sudamérica. Representa un artículo de reflexión.

2 Ingeniero Financiero, Universidad Católica Boliviana "San Pablo", Cochabamba, Bolivia. Consultor en Rediseño Curricular y Docente Pregrado, Universidad Católica Boliviana "San Pablo", Cochabamba, Bolivia. Asesor financiero. Miembro del Comité Científico de la Revista "Ingeniería Industrial: Actualidad y Nuevas Tendencias" de la Universidad de Carabobo, Venezuela. Dirección postal: Calle M. Márquez esq. Parque Trigo Andia, Cochabamba, Bolivia. Correo Electrónico: jairocespedeslopez@gmail.com

(C) Los autores. Este artículo es publicado por Pensamiento Crítico de la Facultad de Ciencias Económicas, Universidad Nacional Mayor de San Marcos. Este es un artículo de acceso abierto, distribuido bajo los términos de la licencia Creative Commons Atribucion - No Comercia_Compartir Igual 4.0 Internacional. (http://creativecommons.org/licenses/by-nc$\mathrm{sa} / 4.0 /$ ) que permite el uso no comercial, distribución y reproducción en cualquier medio, siempre que la obra original sea debidamente citada. 


\section{ABSTRACT}

Financial education is an important factor in the life of any person, consumption, savings, investment and other basic concepts of finance are unknown by much of the population in the world, this article identifies certain attitudes against it and proposes, in a basic way, fundamental pillars of financial education that could be implemented in secondary education, considering that it should be implemented as a curricular subject in education.

Keywords: Financial education; resource management; finance.

\section{Introducción}

En la actualidad existen muchos programas de educación financiera para todo tipo de entes, programas para universitarios, profesionales, programas dedicados a comerciantes o miembros de alguna institución que capacita a las personas o entidades que forman parte de él, sin embargo la educación financiera no está implementada de manera curricular en el nivel de formación secundario de los jóvenes, por lo cual esto se convierte en una necesidad de estudiar más a profundidad en cualquier sociedad este fenómeno, haciendo énfasis en este artículo a Bolivia y Sudamérica.

De esta manera se realiza un análisis en base a datos existentes y posteriormente un análisis cuantitativo en base a opiniones de personas de distintos países del continente y ante la previa identificación de las bases que debería tener la educación financiera en un nivel colegial de formación secundario, en base a que la educación financiera no es un concepto de un solo país, sino de la población mundial en general.

Es casi una obligación realizar estudios en base a la implementación de la educación financiera de manera curricular para los jóvenes preuniversitarios, para que se realice una profundización en el tema, que puede ser aspecto de análisis cuantitativo y cualitativo desde distintos puntos de vista, lo que el presente artículo pretende es dar a conocer que existe una necesidad fehaciente de profundizar en esta disciplina.

\section{Educación financiera: Comprensión global}

Los programas de educación financiera tanto en Bolivia como en muchos países no están incluidos curricularmente en la formación de los jóvenes, es decir, aquellos que cursan el nivel secundario en la etapa todavía colegial de formación académica. 
Si bien existen proyectos, clases particulares, capacitaciones y demás actividades relativas al tema central, estas no cumplen con el estándar de una educación básica financiera para los estudiantes de nivel secundario.

El dilema se encuentra en la etapa en la que se deben manejar las capacitaciones financieras, donde la educación financiera se puede desarrollar en cursos de larga duración, porque consta de muchos conceptos allegados netamente a las finanzas, las inversiones, productos y servicios financieros, medios de pago y seguros, entre otros.

En realidad el concepto de educación financiera según la OCDE (2005) es considerada como:

"el proceso por el cual los consumidores/inversionistas financieros mejoran su comprensión de los productos financieros, los conceptos y los riesgos, $y$, a través de información, instrucción y/o el asesoramiento objetivo, desarrollan las habilidades y confianza para ser más conscientes de los riesgos y oportunidades financieras, tomar decisiones informadas, saber a dónde ir para obtener ayuda y ejercer cualquier acción eficaz para mejorar su bienestar económico".

A su vez, la Corporación Andina de Fomento (2013, p.15) afirma que "la educación financiera puede empoderar a las personas al permitirles administrar de mejor manera sus recursos y las finanzas de sus familias. En América Latina, esto puede ser aplicado tanto a la creciente clase media, que podría necesitar manejar de mejor manera sus finanzas en el largo plazo, así como a las personas de bajos ingresos o a los sectores que se encuentran financieramente excluidos."

De esta manera es importante recalcar que la educación financiera desde esa perspectiva coadyuvaría en su totalidad a la formación de los jóvenes que cursan el nivel secundario de su formación. Si bien la educación financiera es un beneficio para cada persona y por supuesto para cada familia, la Corporación Andina de Fomento (2013, p.16) indica que:

Los beneficios de la educación financiera también pueden extenderse a la economía en general. La educación financiera puede promover las competencias necesarias para tomar decisiones informadas y apropiadas, así como proporcionar herramientas para que las personas tengan la capacidad de defender sus derechos como consumidores finan- 
cieros. Por otra parte, los ciudadanos financieramente alfabetizados tendrán una mayor capacidad para comprender las políticas económicas y sociales adoptadas en sus economías.

La educación financiera entonces es un pilar fundamental para la gestión y uso de capitales, y para un entendimiento más aproximado de la manera en la que los distintos entes que componen un país gestionan sus recursos financieros.

\section{Experiencia en España}

En Europa, uno de los países que ha implementado un programa de educación financiera a la educación del nivel secundario es España, que posee incluso un documento que titula Educación Financiera en Ia Enseñanza Secundaria Obligatoria, mismo que es un texto base para el desarrollo de este programa, cabe resaltar que en el texto indica que es una guía para el alumno y a su vez se tiene un texto guía para el profesorado.

Esto quiere decir que se ha realizado un arduo trabajo en función a este cometido, aspecto no menos importante, puesto que este tipo de educación para jóvenes de esa edad es realmente significativo y puede marcar un antes y un después en su vida.

Esta iniciativa en la educación secundaria de España, ha sido formulada por el Ministerio de Educación, el Banco de España y la Comisión Nacional del Mercado de Valores para la Educación Financiera en España. Claramente no es un trabajo fácil, puesto que se ha formulado el programa a través de distintas instituciones, pero es sin duda necesario.

Al respecto el Ministerio de Educación de España, el Banco de España y La Comisión Nacional del Mercado de Valores (2010, p.1) plantean los siguientes temas como fundamentales para la educación financiera en el nivel de formación secundario, "educación y seguridad financiera, el consumo inteligente, el ahorro, el presupuesto personal, el dinero en efectivo, las cuentas bancarias, las tarjetas, protección de los datos personales, relaciones bancarias"

Temas absolutamente adecuados para la inclusión de la educación financiera en la educación secundaria de cualquier país, sería totalmente pertinente realizar un análisis adecuado de la cultura financiera en cualquier sociedad, su realidad, su proyección, sus visiones, sus leyes, 
su sistema financiero, sus costos y precios, entre otros conceptos que se podrían estudiar y analizar.

\section{Analfabetización financiera en el mundo y en Bolivia}

S\&P Ratings (2015) indica que los países que tienen más desarrollo de la cultura y alfabetización financiera son Australia, Canadá, Dinamarca, Finlandia, Alemania, Israel, Holanda, Noruega, Suecia y el Reino Unido, de estos es importante mencionar que este estudio que afirma esto se ha realizado solamente a personas mayores de edad.

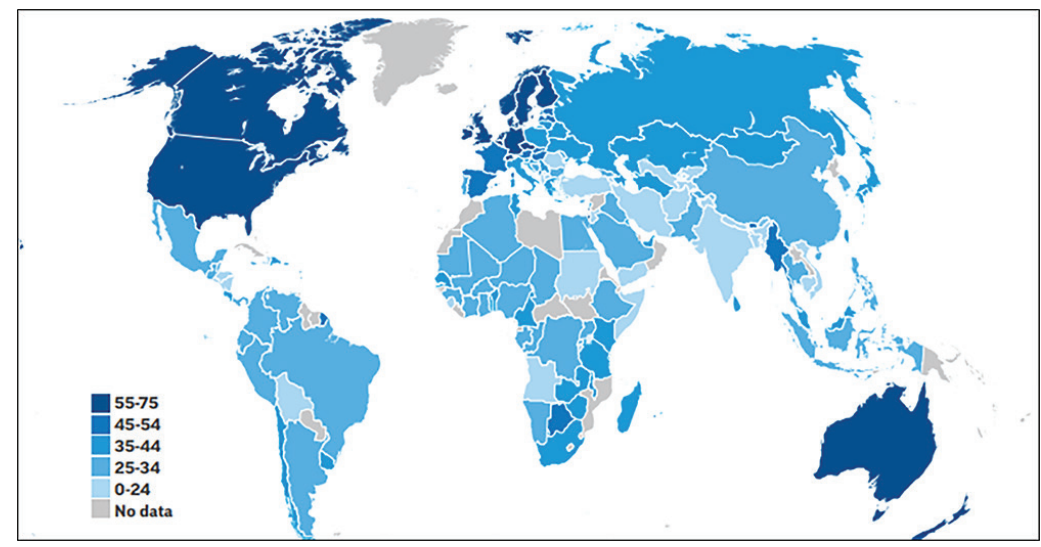

Gráfico 1. Grado de educación financiera en el mundo Fuente: Standard \&Poors Ratings, 2015

En el gráfico 1 se observa claramente que los países de Sudamérica se encuentran en un nivel medio, comprendiendo la escala que se encuentra en el lado izquierdo inferior de la ilustración como el nivel de porcentaje de personas mayores que se encuentran alfabetizados financieramente.

En Sudamérica, gran parte de los países se encuentran entre el 25\% y $34 \%$, mientras que la menor proporción de países se encuentra entre el $35 \%$ y $44 \%$.

Por otro lado, Bolivia es el país con menor nivel de alfabetización financiera en el continente, lo cual representa un problema cuando se quiere o se pretende realizar una educación financiera, puesto que la población mayor de edad no tiene una base en este campo, y si la tiene es un porcentaje menor. 
Dentro de los países con mayor alfabetización financiera se encuentran Chile y Uruguay, considerando solamente la población mayor de edad como demuestra la estadística.

Así mismo, Asobancaria (2016) argumenta que se evidencia que en América Latina no existe un apego educacional en cuanto a las finanzas, ni siquiera en su manera básica, de forma que aspectos tales como inflación, tasa de interés, relación beneficio costo y/o rentabilidad no son conocidos por la población, hecho que hace que la inclusión financiera se dificulte.

De la misma forma, Asobancaria (2016) indica que los niveles de alfabetización financiera necesitan de una medición, esto para que antes de implementarse cualquier estrategia educacional académica de formación, se evalúen los detalles y características que estos deben tener debido a que este estudio representa una base para determinar los temas que son necesarios abordar en cuanto a la educación financiera.

A pesar de todo lo que se ha mencionado anteriormente, el panorama en Latinoamérica ha ido mejorando, en función a los proyectos que se han presentado en los distintos países, en relación a los cursos que existen de educación financiera.

En Latinoamérica Asobancaria (2016) indica que existen tres países que se han destacado sobre todos los demás, estos son Brasil, México y Perú, donde existen cooperaciones interinstitucionales, que se dan usualmente entre el sector público y el sector privado, lo cual hace que el trabajo que se realiza sea más fuerte en cuanto a riqueza académica y credibilidad ante la sociedad.

\section{Inclusión financiera en el nivel de formación secundario}

La analfabetización financiera, o alfabetización financiera, del modo que quiera llamarse, no tiene diferencia alguna, puesto que las corrientes que se han manejado las últimas dos décadas en Latinoamérica apuntan hacia el mismo horizonte, el fin es generar una educación financiera que coadyuve a que la población de cada uno de los países, y cada uno de sus habitantes desarrollen sin dificultad sus habilidades en el ámbito de las finanzas, distribuyéndose esto en distintos campos. 
En cualquier país en el que no se tenga una formación siquiera básica en las finanzas van a existir problemas de diversa índole, lo cual genera entre otras cosas algunos de los siguientes fenómenos:

- Ignorancia de las formas de ahorro más eficientes

- Ignorancia sobre las alternativas de inversión más peculiares

- Pérdida de dinero por falta de información fidedigna

- Adquisición de préstamos de entidades no reguladas

- Pérdidas de alternativas de estudios en centros de estudios privados

\section{Medición de capacidades financieras}

El informe presentado por la Superintendencia de Bancos e Instituciones Financieras de Chile realiza una medición sobre las capacidades financieras de algunos países de Sudamérica. Esta consta a su vez del estudio capacidades en función a índices de educación financiera, mismos que son: conocimiento, comportamiento y actitud en este ámbito.

Tabla 1.

Puntajes de educación financiera - comparativa andina

\begin{tabular}{lcccc}
\hline País & Conocimiento & Comportamiento & Actitud & $\begin{array}{c}\text { Educación } \\
\text { Financiera }\end{array}$ \\
\hline Chile & 5,12 & 5,83 & 3,03 & 13,98 \\
Bolivia & 4,83 & 5,39 & 3,55 & 13,80 \\
Colombia & 5,13 & 5,15 & 3,32 & 13,60 \\
Ecuador & 5,08 & 5,14 & 3,23 & 13,50 \\
Perú & 4,58 & 4,68 & 3,60 & 12,90 \\
\hline
\end{tabular}

Fuente: SBIF en base a CAF, 2016

Es preciso mencionar que el conocimiento financiero consta de preguntas relacionadas con conceptos financieros y fórmulas matemáticas básicas, aspecto en el que Colombia lidera el ranking con un puntaje de 5,13, seguido de Chile, Ecuador, Bolivia y Perú.

Por otro lado, el comportamiento representa la tenencia y utilización de un presupuesto, el manejo de los recursos financieros, comportamiento 
de ahorro y la forma de elección de productos financieros en cada uno de estos países, donde lidera claramente Chile, seguido de Bolivia, Colombia, Ecuador y por ultimo Perú.

Por último, la actitud financiera está compuesta por cinco niveles de preguntas relacionadas a la actitud que las personas poseen en la utilización del dinero, ranking en el que lidera Perú, seguido de Bolivia, Colombia, Ecuador y Chile. En este, aquellos que tienen menor puntaje son los que planifican financieramente a largo plazo, por lo cual Chile se encuentra en una mejor ubicación. En cuanto a la educación financiera, Chile lidera el ranking, situación que es el resultado del cálculo matemático de los tres aspectos anteriormente mencionados.

\section{La necesidad de la educación financiera en la formación académica preuniversitaria y su estructura básica}

La educación financiera en el currículo académico en el nivel secundario, colegio, escuela, secundario, educación media o cualquier otra denominación que tenga en los diferentes países, es todavía un deseo de muchos y un aspecto desconocido por otros, sin embargo la aplicación de cualquier encuesta relacionada al tema sería totalmente redundante, seria completamente vana considerando que este fenómeno ha sido muchas veces puesto en la consideración popular y siempre ha sido solamente punto de atención a las mediciones de valor.

La Cooperación Andina de Fomento (2013) indica que según los lineamientos que plantea la INFE/OECD

"La educación financiera debe ser integrada en los currículos escolares como parte de una estrategia nacional coordinada más amplia que involucre a la comunidad, debe tener como audiencia objetivo a todos los niños y niñas dentro de su jurisdicción y debe estar precedida por un estudio del estado y el nivel de la alfabetización financiera de los jóvenes. También recomiendan involucrar al Ministerio de Educación y a las entidades educativas interesadas. Asimismo, los lineamientos sugieren el uso de modalidades flexibles, que puedan adaptarse a las circunstancias nacionales y locales."

El concepto correcto para la educación financiera en un currículo académico de formación pre universitaria seria "inclusión", de esta 
manera estamos hablando entonces de "inclusión financiera", es que se considera a la educación financiera como una rama de las finanzas y la administración como una ciencia difícil de estudiar en poco tiempo.

En ese sentido, es preciso definir la palabra "inclusión", que según la Real Academia Española significa lo siguiente:

- Acción y efecto de incluir.

- Conexión o amistad de alguien con otra persona.

Tal como se ve en estas definiciones, la educación financiera debe ser una manera de inclusión para aquellas personas que se encuentran en un periodo de formación crucial, previo a la educación pre gradual, además de significar un boleto a la evasión de distintos aspectos de fraude o simplemente aspectos cotidianos tales como:

- Estafas en venta de terrenos.

- Estafas en venta de productos o servicios.

- Selección de instrumentos financieros.

- Selección de bancos para la apertura de cuentas bancarias.

- Inversiones por internet.

- Estafas en internet.

- Compras por internet.

- Utilización de la tarjeta de debido y de crédito.

- Ahorro.

- Compras compulsivas.

El punto es que la inclusión financiera para estudiantes de colegio que se encuentren en el nivel de formación secundario debería contener los siguientes aspectos en opinión de académicos del área.

- El consumo

- El ahorro

- La inversión

- Presupuesto personal

- Presupuesto familiar

- Dinero en efectivo

- Dinero virtual

- Bancos

- Cuentas bancarias 
- Tarjetas de debito

- Tarjetas de crédito

- Compras por internet

- Seguridad de los datos personales

- Estafas piramidales

Para esto debe existir una planificación presupuestaria en el ámbito de la educación y sobretodo una planificación organizacional, aspecto en el cual deberían participar las grandes instituciones financieras de cada país y obviamente el ministerio de educación y las grandes entidades públicas decisionales.

\section{Factores determinantes ante una posible implementación de la educación financiera en el currículo de la formación secundaria.}

La implementación de este concepto bajo modalidad de materia curricular representaría al estado costos adicionales por los siguientes factores:

\section{Tabla 2.}

Gastos inherentes del gobierno en caso de implementar la educación financiera en la educación secundaria

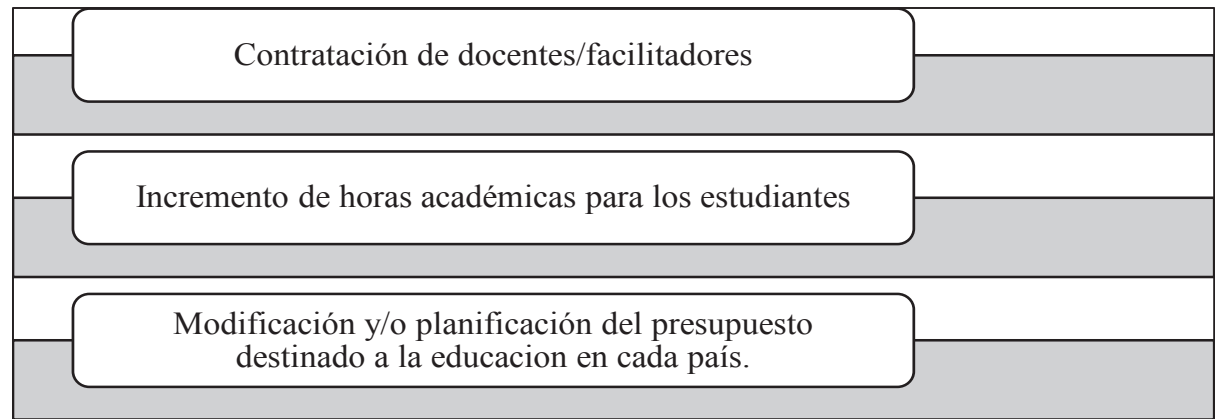

Fuente: Elaboración propia en base a revisión bibliográfica.

Bajo estos principios, es necesario hacer énfasis en la importancia sobre el Producto Interno Bruto (PIB) que cada uno de los países de Sudamérica le da a la educación de forma genérica. En el siguiente cuadro se percibe lo anteriormente mencionado: 


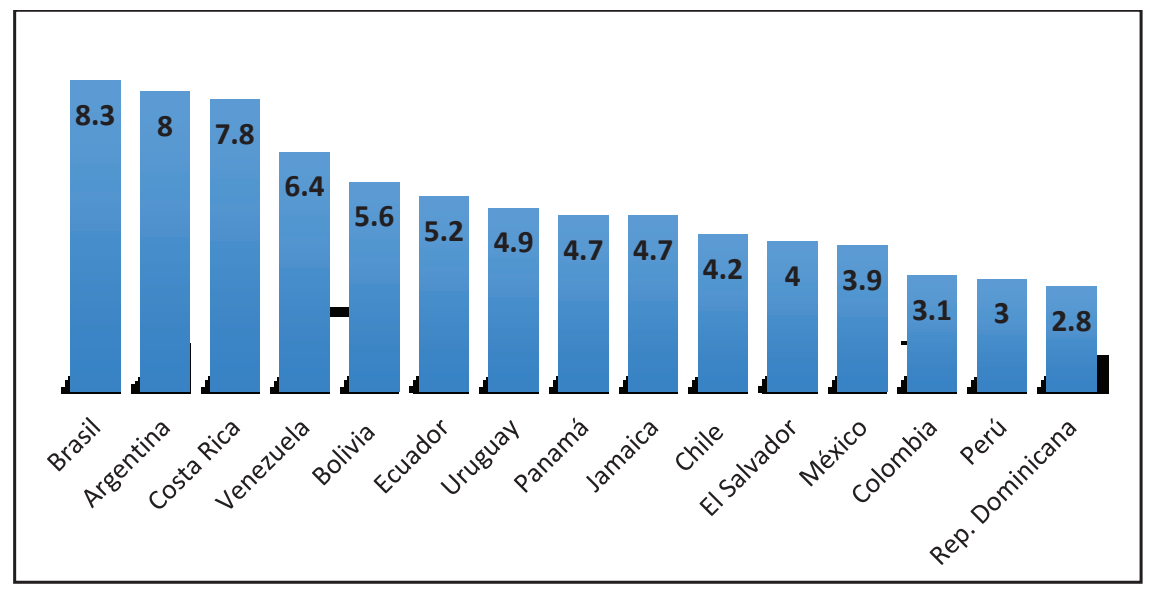

Gráfico 2. Porcentaje del PIB destinado a la Educación Financiera

Fuente: Elaboración propia en base a Informe Pulso Social de América Latina y el Caribe 2016

Se puede ver entonces, que el país que utiliza más presupuesto haciendo una comparación con el PIB de su país es Brasil, con un 8,3\% y de manera contraria, República Dominicana 2,8\% de su PIB lo destinan a la educación.

El estado dentro de cada país es aquel que distribuye los recursos a las provincias, departamentos, localidad eso como se denominen, por lo cual, una mejora en la educación es casi una obligación, por lo que su presupuesto anual está completamente sujeto a una planificación y ante la posible implementación de la educación financiera de manera curricular en cualquier país deben considerarse los gastos adicionales mencionados en la tabla 2.

Por otro lado, los cambios considerables se presentarían en la economía, el sector comercial y financiero de los países, a través de la actitud que las personas tomarían con un conocimiento financiero aunque este sea básico, puesto que existiría mayor movimiento en distintos campos de la economía.

Otro aspecto a mencionar es que la educación financiera no es un punto que pasa desapercibido para los jóvenes que cursan dicho nivel, es 
decir jóvenes de entre 13 a 17 años aproximadamente dependiendo de los países y su estructura de la educación secundaria.

Considerando que los jóvenes son completamente despiertos a temas de carácter actual y tecnológico es preciso afirmar que la formación en aspectos de carácter financiero no será en vano para ellos, siempre tratarán de poner en práctica sus conocimientos.

Análisis cuantitativo sobre la percepción de las personas en la situación actual en función a su capacidad de administración de recursos y características del ámbito tecnológico financiero

Para este análisis se ha realizado una encuesta a jóvenes colegiales, universitarios y personas mayores en base a su cultura financiera actual y en base a los pilares fundamentales identificados anteriormente; esta se ha realizado a personas de distintos países de Sudamérica, enfocándose de esta manera en la idea de que la educación financiera va más allá de las fronteras y que debería ser una doctrina mundial desarrollada.

Haciendo énfasis en la actualidad, las personas no tienen una formación en educación financiera en ningún momento de su vida, ni en la educación básica ni secundaria; y por otro lado los jóvenes universitarios conocen del tema a través de sus carreras, es decir, aquellos que estudian carreras relacionadas con las ciencias administrativas de cierta forma asimilan los aspectos más determinantes de la educación financiera, pero esto es subjetivo, porque bien sabemos que la educación financiera puede ser estudiada a lo largo de muchos años, incluso podría ser una carrera universitaria, o algún tipo de formación técnica en el área.

De esta manera, se encuentra solo un segmento de la población dentro de un conocimiento básico, pues no es a total profundidad, existen pocas carreras universitarias que realmente podrían solventar este problema, por ejemplo la carrera de Ingeniería Financiera, Finanzas, Administración Financiera y otras relacionadas al ámbito de las finanzas, que realmente tocan aspectos netos de la educación financiera en distintos lugares tanto de Sudamérica y el mundo.

Por otro lado, incluso profesionales formados en otras áreas de estudio y desempeño no conocen del tema, estudiantes de colegio recientemente graduados no conocen de la educación financiera, lo cual 
provoca una analfabetización en este sector, que puede causar muchos problemas financieros tanto personales como familiares en el futuro.

En ese sentido se ha realizado una encuesta a personas de distintos países, a continuación en el gráfico 3 se muestra la condición de la persona que ha sido encuestada.

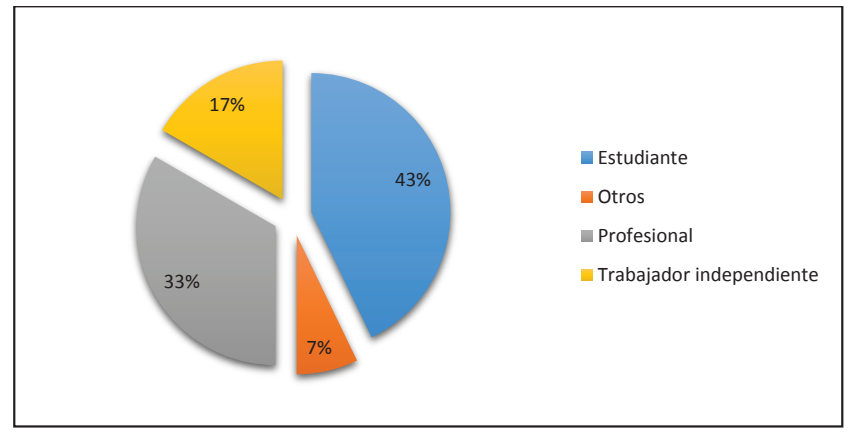

Gráfico 3. Generación de ingresos según su ocupación

Fuente: Elaboración propia en base a los resultados de la encuesta sobre cultura financiera, 2017

En su mayoría los encuestados fueron estudiantes y profesionales, cabe resaltar que con otros se hace referencia a personas que no cuentan con una fuente laboral fija y que pueden realizar actividades por ejemplo de manualidades, venta de comida y otros. Este es un buen parámetro porque se analizan los resultados sobre todo desde dos dimensiones, quizá tres incluyendo a los trabajadores independientes, que pueden ser consultores en distintas áreas, importadores, comerciantes, y otros de distintos rubros.

En base a los pilares que se han identificado anteriormente se analizan los resultados.

\subsection{Ahorro}

El ahorro es uno de los factores que más variarían en función a una aplicación de este concepto mucho más efectivo y detallado en relación a lo enseñado y aprendido por los estudiantes.

BAC CREDOMATIC (2008, p.195) indica que el ahorro es la diferencia que existe entre el ingreso y el consumo de una persona, una familia, una 
institución o un país, considerando a este como uno de los mejores hábitos que pueden tener las personas.

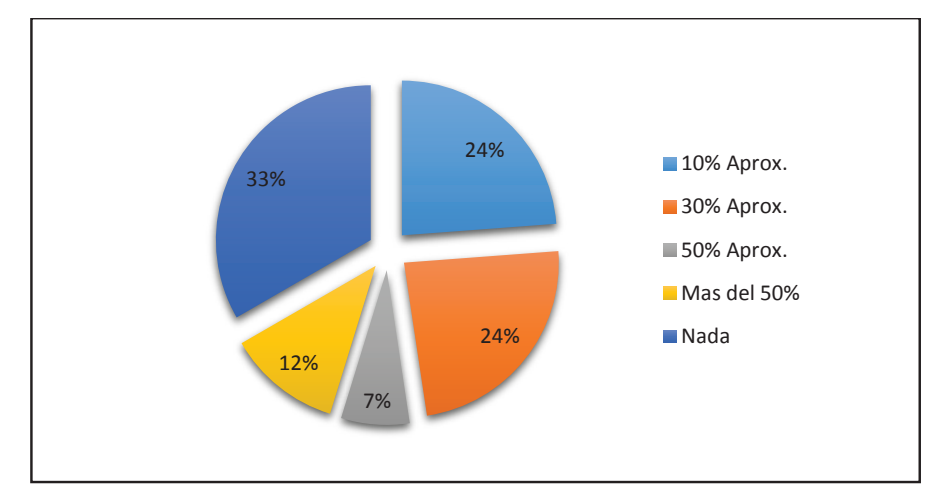

Gráfico 4. Nivel de ahorro en las personas

Fuente: Elaboración propia en base a los resultados de la encuesta sobre cultura financiera, 2017

Como se puede apreciar en el Gráfico número 4, el 33\% de las personas en la actualidad no ahorran nada de sus ingresos, entendiendo ingresos como sueldos, salarios, mesadas y cualquier otro concepto que genere la atracción de recursos monetarios para el uso propio de la persona, aspecto que indica la falta de cultura financiera.

Esto determina claramente que la educación financiera es un punto determinante a tener enfocado para su cuidado y perfeccionamiento en cada país; por otro lado un 30\% afirma que ahorra aproximadamente un $10 \%$ y un $30 \%$ con el mismo valor, cabe aclarar que ahorrar cualquier monto de dinero es totalmente valorable, pero es preocupante conocer este tipo de cifras en la sociedad.

Por último un 12\% afirma que ahorran más del 50\% y un 7\% ahorra el $50 \%$ de sus ingresos, lo cual es un porcentaje mínimo de personas que tienen un grado de cultura financiera básica puesta en práctica en cuanto al ahorro.

Dentro del ahorro existen muchos mitos, contradicciones y mentiras, algunas personas afirman que el ahorro es una pérdida de tiempo porque es dinero que está estático y no genera retribución alguna, sin embargo existen diversos motivos por los cuales la gente ahorra, entre los cuales se encuentran por ejemplo los siguientes: 
- Comprar un vehículo

- Viajar

- Pago de estudios

- Comprar artefactos para el hogar y otros.

Si bien se puede considerar el pago de estudios como una inversión a futuro, es preciso entender este como un ahorro, dado que el simple hecho de guardar dinero para posteriormente utilizarlo en alguna actividad representa un ahorro, lo que posteriormente suceda puede o no ser una inversión.

\subsection{Inversión}

La inversión es considerada como aquel monto de dinero puesto en marcha en alguna actividad que dentro de un tiempo generará retribución financiera, es importante tener un grado de inversión en el cotidiano vivir, puesto que le otorga al tenedor de dinero cierta independencia en cuanto a la administración de sus recursos, por más de que una persona perciba un sueldo alto, es preciso mencionar que mientras más uno tiene, más uno gasta, el gasto estádirectamente relacionado con el nivel de ingresos.

Pocos son lo que realmente invierten su dinero, incluso aquellas personas que tienen bajos ingresos han hecho inversiones que a la larga resultaron grandiosas y muy fructíferas, la base de esta afirmación es la siguiente gráfica que refleja si los profesionales, estudiantes, trabajadores independientes o personas que generan ingresos de otra manera han invertido alguna vez su dinero, es importante mencionar que solo consideramos la inversión, no así el gasto.

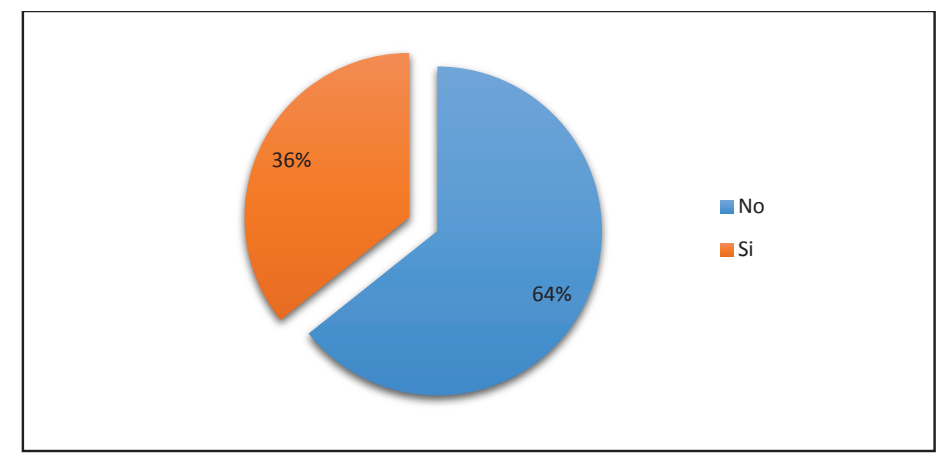

Gráfico 5. Porcentaje de personas que han invertido alguna vez su dinero Fuente: Elaboración propia en base a los resultados de la encuesta sobre cultura financiera, 2017 
Como se puede apreciar en el gráfico 5, el 64\% de las personas nunca han invertido su dinero, por otro lado, el 36\% de las personas si han realizado esta operación, es curioso que solamente este porcentaje haya hecho uso de este concepto tan importante, puesto que es parte fundamental de la educación financiera.

Si por ejemplo, se ahorra toda la vida, se tendrá seguramente mucho dinero para la vejez, dinero que con seguridad ha de disfrutar en plenitud, o repartirá dicho dinero entre todos los hijos, que es lo que usualmente hacen las personas de la tercera edad.

En otro escenario se tendría el solo hecho de ahorrar el dinero y que algún momento este se haya invertido, y que este haya generado réditos financieros importantes a lo largo de muchos años, es más que seguro que las personas que han invertido su dinero tendrán aún más riquezas acumuladas que las personas que solo han ahorrado.

El gasto a su vez forma parte de la inversión, invertir no significa no gastar, tanto las personas que ahorran como las personas que invierten tienen gastos.

El principio de la inversión, tal y como menciona BAC CREDOMATIC (2008, p. 132), afirma que sin inversión es difícil, si no imposible tener algún éxito financiero, este no se adquiere con el ahorro y si esto sucede demora mucho más tiempo, por lo cual es preciso tomar ciertos riesgos en las inversiones.

\subsection{Presupuesto}

El presupuesto es el cálculo anticipado de ingresos y gastos para un periodo de tiempo futuro, tanto de manera personal como familiar; BAC CREDOMATIC (2008, p.166) indica que es importante que un presupuesto contenga metas financieras, a corto, mediano y largo plazo, mismas que deben ser específicas, medibles, alcanzables y poseer un marco de tiempo establecido y especifico. Lo ideal sería que por lo menos el $90 \%$ de las personas cuenten con un presupuesto, obviamente aquellas con reciben ingresos de cualquier índole. 


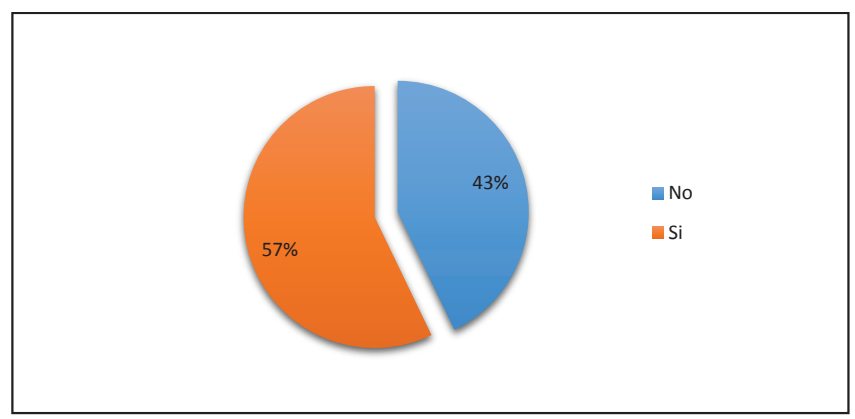

Gráfico 6. Porcentaje de personas que cuentan con un presupuesto personal Fuente: Elaboración propia en base a los resultados de la encuesta sobre cultura financiera, 2017

Tal y como se puede percibir en el gráfico 6, el 57\% de las personas cuentan con un presupuesto personal, lo cual es aún un porcentaje bajo en relación a lo que realmente sería importante, eso quiere decir que el 43\% no hacen cuentas frente a sus gastos futuros, también quiere decir que estas se encuentran más propensas a tener problemas relacionados con el dinero en su vida personal.

El contar con un presupuesto personal es significativo para cualquier ciudadano, considerando que existen aquellos que cuentan con deudas, y que de alguna manera deben prever sus cuentas, calcular sus ingresos y sus gastos, o considerando que existen personas que deben realizar pagos de diversa índole, estos deben considerar todos sus movimientos financieros ya sea de manera mensual, bimensual, semestral o en cualquier periodo de tiempo.

Por ende, el presupuesto personal es de vital importancia, por otro lado se encuentra el presupuesto familiar, que es sobre todo para aquellas personas que están casadas; es innegable considerar que existen diversas situaciones familiares, en función al número de hijos, ingresos o por consiguiente al nivel de gastos.

En cuanto al presupuesto familiar sucede lo siguiente. 


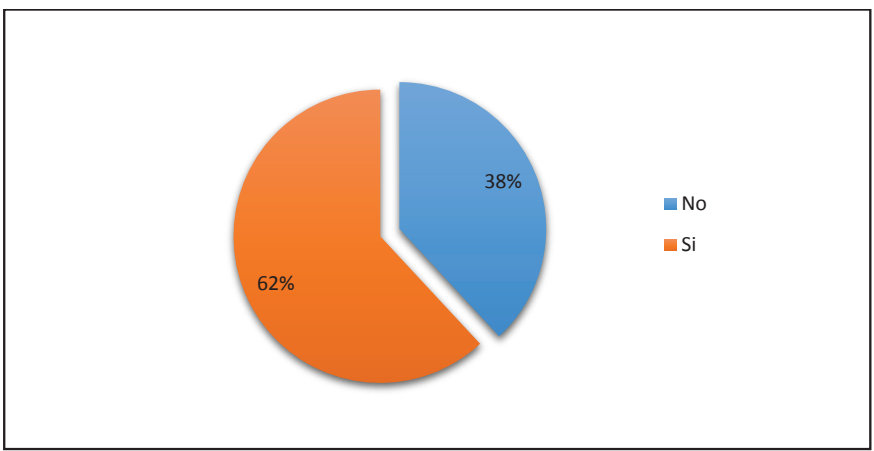

Gráfico 7. Porcentaje de familias que cuentan con un presupuesto familiar Fuente: Elaboración propia en base a los resultados de la encuesta sobre cultura financiera, 2017

Un $62 \%$ de las familias han respondido que cuentan con un presupuesto, lo cual es muy importante para la economía en cada país, en este aspecto también debe considerarse que existen diversos tipos de familias, y distintos tipos de clases sociales, con niveles de ingresos diferentes, sin embargo es un porcentaje importante. El presupuesto familiar a su vez es un aviso a futuros problemas monetarios, se pueden considerar problemas tales como la presentación de algún suceso inesperado, algún gasto que no estaba previsto, la cuestión es, si debería existir la casilla o la cuenta de imprevistos dentro de un presupuesto familiar.

La respuesta a esta cuestionante es complemente afirmativa, la existencia de una cuenta de imprevistos es tan importante como la cuenta de gastos o la cuenta de ingresos, debido a que cualquier hecho que pueda suceder hace variar completamente el presupuesto si este ítem no está incluido.

El presupuesto familiar es uno de los puntos relativamente altos dentro de la educación financiera actualsin la implementación de esta como materia curricular dentro de la educación secundaria, es de cierta forma un punto a salvar dentro del conocimiento y aplicación de las finanzas básicas dentro de la vida familiar, no así personal.

\subsection{Cuenta bancaria}

La existencia de una cuenta bancaria en la actualidad representa un factor importante en la vida de las personas, tener una cuenta bancaria en la 
institución financiera de su elección le otorga responsabilidad, seguridad y sobretodo accesibilidad a diversas formas de pago, así como negociar a través de internet de acuerdo a las facilidades que le otorgue dicha institución.

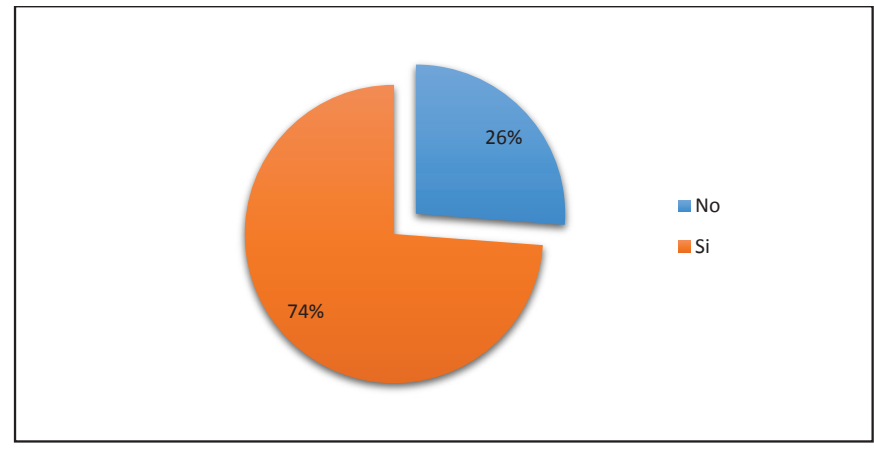

Gráfico 8. Porcentaje de personas que poseen una cuenta bancaria

Fuente: Elaboración propia en base a los resultados de la encuesta sobre cultura financiera, 2017

Tal y como muestra el gráfico 8 , el $74 \%$ de los encuestados ha indicado que poseen una cuenta bancaria, a primera vista este es un factor importante e indica que la cultura financiera no esta tan descuidada como parece en algunos aspectos anteriores, sin embargo en el aspecto transaccional sucede lo contrario, todo lo que parece ser cuasi perfecto se torna diferente en base al siguiente gráfico.

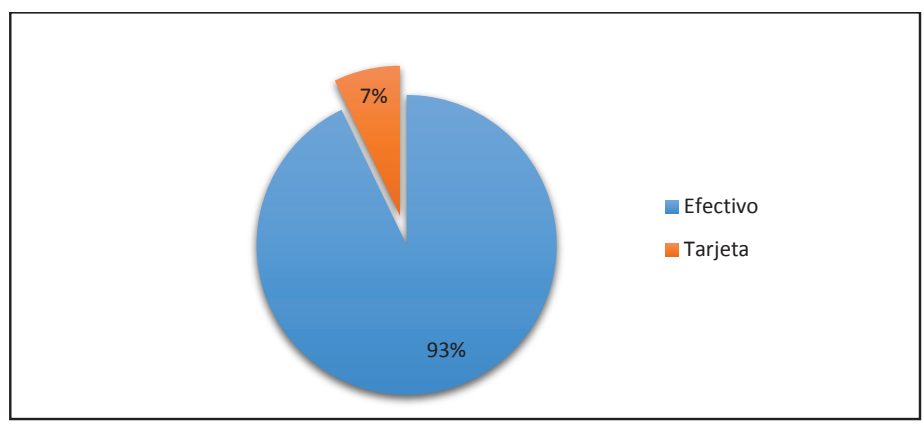

Gráfico 9. Uso de efectivo o tarjeta para pago de transacciones

Fuente: Elaboración propia en base a los resultados de la encuesta sobre cultura financiera, 2017

El 93\% de las personas hacen uso de efectivo para pagar sus transacciones en cualquier punto de venta, considerando que hoy en 
día gran parte de los negocios poseen sistemas de pago electrónicos, es preocupante que tan solo el 7\% haga uso de este servicio.

El uso de tecnología financiera es sin duda un aspecto que debería ser profundizado en el manejo de las finanzas personales e interpersonales, la tarjeta bancaria y su uso para realizar transacciones debe ser mucho más palpable en la sociedad, el hecho de tener que sacar dinero en efectivo para pagar cualquier tipo de transacción quiere decir que la gente aún tiene temor o desconfianza con respecto a la tecnología en las finanzas, lo que desgasta su cultura y educación financiera.

En cuanto a los productos y servicios financieros estos deben ser más accesibles y esto se logra a través de una mayor oferta y facilidad de utilización, este aspecto incrementaría sin duda la educación financiera de la ciudadanía, lo cual también sería bienvenido en cuanto a las transacciones virtuales.

\subsection{Compras por internet}

La cultura financiera a su vez, a nivel de educación debe tener en cuenta diversos factores, y en la actualidad no se puede dejar atrás la tecnología y el uso del internet como una de las herramientas básicas pero sin embargo fundamentales.

BAC CREDOMATIC (2008), indica que comprar es "un intercambio de dinero, por objetos, mercancías y servicios, adquirimos lo que requerimos por para nuestra subsistencia"

De esta manera se debe adaptar este concepto a las operaciones virtuales, en las que se hace lo mismo a través de un pago electrónico, que representa otro medio de pago, que de igual manera BAC CREDOMATIC (2008), muestra que los medios de pago deben tener facilidad de uso, universalidad, liquidez del instrumento, fraccionamiento, seguridad y garantía.

Por otro lado, se ha determinado que muchas personas poseen una cuenta bancaria, sin embargo muchas de estas siguen pagando con efectivo, una de las razones más fuertes y relevantes es la poca información y la desconfianza ante el uso de la tarjeta como medio de pago, lo cual hace que la cultura financiera disminuya por tal suceso. 
De esta forma, alegando al uso de la tarjeta bancaria, existen muchas instituciones financieras por no decir todas, que ofrecen el servicio de compras por internet, pago de servicios vía internet y contratación de algunas comodidades vía internet a través de sus cuentas bancarias. Sería ilógico que el resultado de esto sea favorable si aún existen personas que por falta de información no hacen uso de su tarjeta para pagar sus servicios, en ese sentido dicha afirmación se comprueba en el siguiente gráfico.

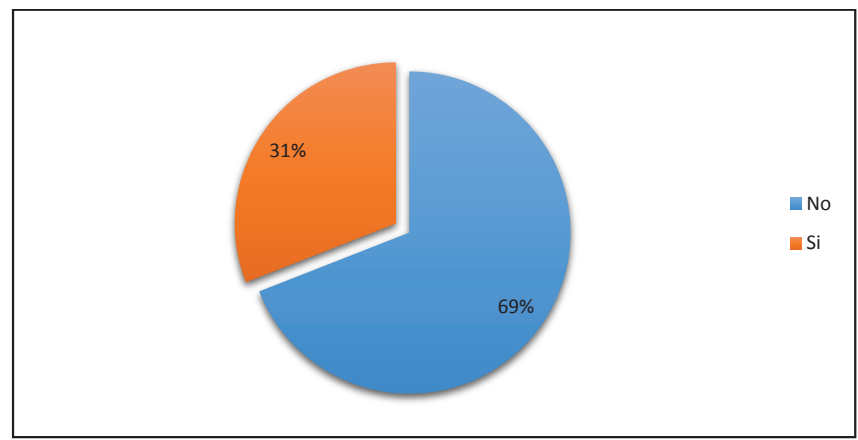

Gráfico 10. Porcentaje de personas que realizan compras por internet Fuente: Elaboración propia en base a los resultados de la encuesta sobre cultura financiera, 2017

Tal y como se ha afirmado anteriormente, solo el 31\% de las personas realizan o han realizado compras alguna vez en internet, esto quiere decir compras via Amazon, AliExpress, E-Bay y otros portales donde se pueden adquirir diversidad de productos.

Mientras otras personas viven de ese negocio, existen otros que no se atreven a navegar en el nuevo mundo de los negocios financieros $y$ transaccionales de productos por falta de información.

Tal cual, a falta de información, muchas personas ingresan a estas páginas, donde deben registrarse y en el momento de hacerlo no llegan a concretar dicha acción por temor, este es sin duda uno de los aspectos fundamentales a mejorar sobre la cultura financiera de las personas.

\subsection{Administración de los recursos}

El tipo de administración de recursos financieros de cada ente representa un factor importante dentro de la cultura financiera, 
sobretodo su percepción acerca de ello, en este sentido todos aquellos que cuentan con recursos ya sean provenientes de un sueldo, salario, mesada o cualquier tipo de ingreso deberían llevar a cabo una administración, la cual obviamente está directamente ligada al uso de un presupuesto, la percepción de las personas de acuerdo a su capacidad de administrar dichos recursos es la siguiente.

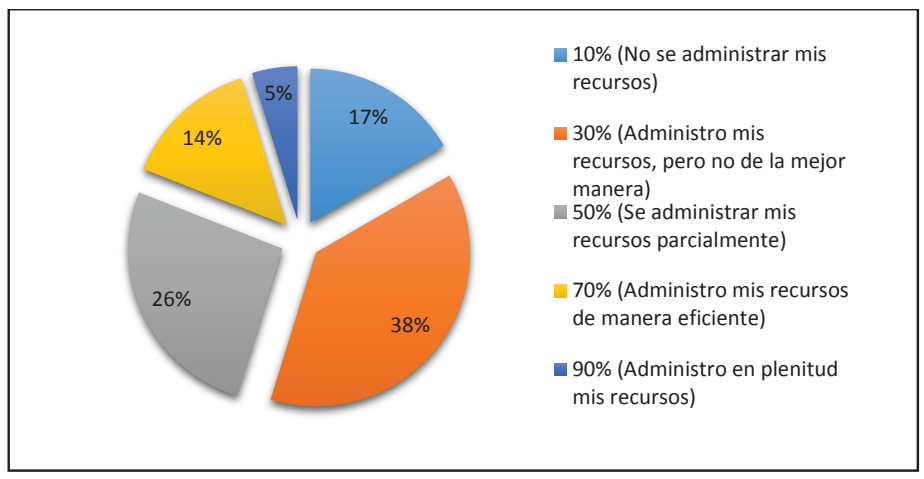

Gráfico 11. Capacidad de las personas en la administración de los recursos Fuente: Elaboración propia en base a los resultados de la encuesta sobre cultura financiera, 2017

De acuerdo al gráfico 11, un 38\% afirma que administra sus recursos pero no lo hace de la mejor manera, lo cual quiere decir que presentan deficiencias ya sean mensuales, semestrales o en cualquier periodo de tiempo, el $26 \%$ de las personas afirman que saben administrar sus recursos de manera parcial, lo cual no está ni entre o malo pero no cumplen al cien por cien con todas sus metas financieras, es más, tanto los primeros como los segundos mencionados con seguridad tienen dificultades en el cumplimiento de sus objetivos.

El 17\% de las personas afirma que no sabe administrar sus recursos, lo cual representa un gran problema en cuanto a su cultura financiera, estas con seguridad tienen deudas aunque mínimas pero las poseen, a su vez tienen dificultad para saldarlas.

Entrando al rango que hace referencia a las personas que administran sus recursos con mayor propiedad, tan solo el $14 \%$ cae en dicha afirmación, estas personas administran sus recursos eficientemente y pocas veces 
necesitan de ayuda económica financiera, las veces que adquieren un préstamo de cualquier índole pueden pagarlo sin ningún problema.

Por último tan solo el 5\% afirma que administra sus recursos de la mejor manera, no tienen problemas financieros, es preciso mencionar que no necesariamente las personas deben tener ingentes cantidades de dinero para gozar de una buena administración, cualquier persona puede lograr dicha meta con un buen conocimiento financiero.

\subsection{Capacitación financiera}

La capacitación financiera se encuentra en proceso de desarrollo en muchos países, si bien existen talleres, cursos y capacitaciones en el área, implementarla formación en este campo de manera curricular a la educación en el nivel secundario sería una excelente forma de profundizar la cultura financiera no solamente de los jóvenes estudiantes, sino de un país entero en el transcurrir del tiempo.

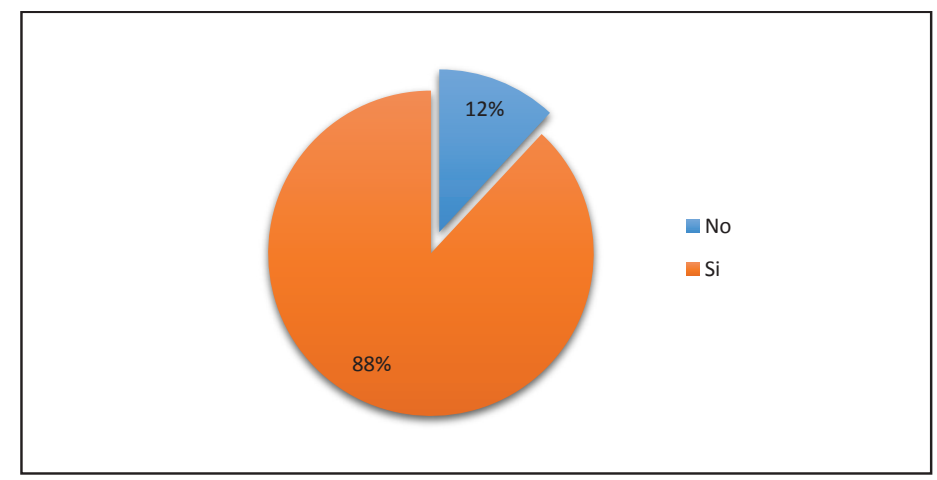

Gráfico 12. Porcentaje de personas que consideran que una capacitación ayudaría en su cultura financiera

Fuente: Elaboración propia en base a los resultados de la encuesta sobre cultura financiera, 2017

Como se observa en el gráfico 12 , el $88 \%$ de las personas ha afirmado que una capacitación en el ámbito financiero coadyuvaría en la administración de sus recursos, hecho que delata que este tipo de procesos en el ámbito de la educación seria en aceptado en gran magnitud sin mencionar que si los jóvenes estudiantes de colegios secundarios llevasen este concepto como materia curricular saldrían totalmente 
preparados para afrontar todas las situaciones que se han mencionado, el ahorro, la inversión, el uso de tarjetas bancarias, pago en efectivo o con tarjeta, compras vía internet y otros.

Según la percepción de estas mismas, una capacitación en el ámbito financiero incrementaría sus conocimientos en los siguientes porcentajes como se observa a continuación:

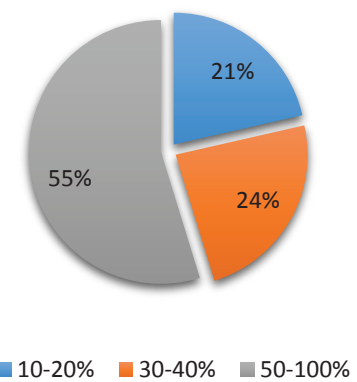

Gráfico 13. Percepción sobre el porcentaje en el que una capacitación financiera ayudaría en la administración de sus recursos.

Fuente: Elaboración propia en base a los resultados de la encuesta sobre cultura financiera, 2017

En el ámbito financiero se deben tener en cuenta muchos aspectos para apoyar a personas que de acuerdo a su experiencia, han creado su propio sistema de administración de recursos financieros, este de manera empírica en función a sus necesidades, muchos ya profesionales, dueños de algún negocio u otra actividad, sin embargo las personas consideran en un $55 \%$ que una capacitación de carácter financiero incrementaría su habilidad de administración de recursos de un 50\% a un $100 \%$, lo cual es totalmente favorable para las finanzas de dichas personas.

Por otro lado un $24 \%$ estima que una capacitación incrementaría su habilidad en un rango de 30\% a 40\%, lo cual aún sigue siendo un número significativo, porque debemos considerar que estas personas ya tienen cierta capacidad de administración, y si la mejoran en tal porcentaje podrían ser eficientes administradores financieros tanto en su vida personal como dentro de sus familias. 
Finalmente un 21\% considera que solo se incrementaría su capacidad de administración en un rango de $10 \%$ a $20 \%$, este conjunto de personas no se ven en el camino de mejorar sus finanzas o de pasar por un proceso de enseñanza que tenga ese propósito.

Como indica la Cooperación Andina de Fomento (2013) "La educación financiera puede promover las competencias necesarias para tomar decisiones informadas y apropiadas, así como proporcionar herramientas para que las personas tengan la capacidad de defender sus derechos como consumidores financieros."

Es así que las capacitaciones en este ámbito contribuyen en gran magnitud a incrementar el conocimiento de las personas en cuanto a los productos y servicios financieros, sus facilidades y ventajas, así como también aspectos negativos para que estos no caigan en estafas de cualquier índole; de acuerdo al estudio y análisis realizado la población encuestada concuerda con que los cursos o capacitaciones en esta área de desempeño coadyuvarían de manera beneficiosa en la administración de sus recursos financieros.

\subsection{Formación en educación financiera en la educación a nivel secundario}

Tal y como se ha mostrado en el análisis anterior, urge la implementación de la educación financiera ya sea de manera curricular o de manera técnica en la educación a nivel secundario de cualquier país.

Si se hace una comparación incrementando los porcentajes en los que la gente considera que una capacitación financiera incrementaría su conocimiento en la administración de sus recursos (gráfico 13) se pueden estimar los resultados de manera aproximada.

Recordemos que un 17\% (escala 10\%) no sabe administrar sus recursos y un 38\% (escala 30\%) administra sus recursos pero no lo hace de la mejor manera, si solo nos centramos en estos dos tipos de personas, y tan solo incrementamos en un $50 \%$ su capacidad, las estadísticas quedarían de la siguiente manera: 
Las personas que no saben administrar sus recursos pasarían a situarse en la escala en un $60 \%$, dentro del rango de las personas que administran su dinero de manera parcial, esto obviamente tomando en cuenta el escenario más pesimista, si se toma el rango más optimista estas personas deberían administrar sus recursos de la mejor manera y sin problemas, lo mismo sucede con los que se situaron en la escala del $30 \%$, si a estos le incrementamos un $50 \%$, se situarían en $80 \%$ y pasarían a administrar sus recursos eficientemente, lo cual sería un avance extraordinario.

Las personas que no han tenido una formación en el ámbito financiero, muchas veces no saben de qué manera administrar su dinero, no tienen la información suficiente para adquirir una tarjeta de débito o no tienen la confianza necesaria para comprar vía internet. En total obviedad, esta capacitación para este tipo de personas que ya han terminado su formación colegial cuesta dinero, lo cual afecta en su economía paradójicamente porque muchos no cuentan con un presupuesto.

La implementación de la educación financiera a nivel secundario significaría un gasto para el estado pero mejoraría la economía de las personas en todo el territorio nacional, vale repetir, de cualquier país.

La formación en el ámbito financiero en jóvenes colegiales incrementaría el tráfico transaccional en el sistema financiero, lo cual hace que la economía crezca, y que el movimiento financiero en los países sea más activo y que este se encuentre en constante proceso de innovación, en cuanto a los productos financieros y demás.

Esta formación no solo afecta en el sector financiero, sino también en el sector comercial, los jóvenes en su calidad de inquietud siempre están atentos a nuevas alternativas de compra, en este caso, la información es un factor fundamental para poder hacer esto posible, cuando se logra la confianza en el cliente o en las personas que deciden emprender algún negocio se ha logrado una fuente de desarrollo nacional.

Sin duda, la educación financiera debe ser planteada como un concepto de inclusión en algunos países de Sudamérica debido a la poca participación de los jóvenes en esta. 


\section{Etapas de la formación financiera y su relación con la educación financiera en los colegios}

Para la educación financiera existen etapas, así lo plantea BAC CREDOMATIC (2008, p.171), quien afirma que son tres, la primera etapa es hasta los 18 años, periodo en el cual se desarrolla el aprendizaje básico financieros, es apropiado mencionar también que durante esta etapa la persona por lo general no percibe ingresos y depende netamente de los padres o tutores. En esta etapa se adquieren la mayoría de paradigmas financieros que a lo largo de la vida serán modelos de comportamiento financiero.

La segunda etapa es de los 18 a los 30 años de edad, etapa que se caracteriza por la formación profesional o técnica o simplemente un oficio, que coadyuvarán a percibir ingresos durante en la vida profesional ganando independencia financiera y de esta manera definir el futuro económico.

Posteriormente se encuentra el periodo entre los 31 y 45 años de edad, que está caracterizado por la conformación de una familia, periodo en el cual los ingresos crecen y por consiguiente también los gastos, se adquieren bienes y ya se crea una independencia financiera en el ámbito familiar, es este periodo una buena administración de los recursos es indispensable, las personas que en este periodo no llevan una cultura financiera adecuada deben capacitarse en el ámbito para no sufrir problemas financieros.

La cuarta etapa está entre los 46 y 55 años, es considerada como la del máximo desarrollo financiero, periodo en el cual se consolida la riqueza familiar, durante este periodo los ingresos crecen hasta llegar a cierto tope.

A los 65 años se inicia el pre-retiro los ingresos decrecen pero la igual que lo mencionado anteriormente decrecen los gastos, debido a que los hijos ya están alcanzando la independencia financiera, lo cual incrementa el ahorro y la inversión sin deteriorar de ninguna manera la calidad de vida de las personas.

Por último después de los 65 años en teoría es la edad del retiro, pero esto solo depende de la formación que se ha tenido en el ámbito financiero. 
En base a las etapas mencionadas, es innegable afirmar que la primera es la más indicada para la formación de los ideales financieros, esta es una afirmación de que la implementación de la educación financiera de manera curricular en colegios seria para los jóvenes una excelente ayuda no solo para esa etapa de su vida, sino para todas las demás también, puesto que como se ha mencionado anteriormente, es la etapa en las cuales una forma sus ideales financieros.

Entonces, es preciso el desarrollo de nuevos programas de educación financiera que tengan como objetivo a los jóvenes que cursan el nivel secundario en colegios de distintos lugares y países del mundo, puesto que es la mejor etapa para la enseñanza de temas financieros.

En base a lo citado y al análisis que se ha realizado, la educación financiera debe ser entendida bajo el concepto de inclusión, y debe ser aplicada de esta manera puesto que en la actualidad existen gran parte de la ciudadanía que no se identifica con este tema, y justamente ese es el fin, reducir ese fenómeno en las próximas generaciones y que estas lleven una vida saludable financieramente hablando.

\section{Conclusiones}

La formación en educación financiera tal y como se ha demostrado a lo largo del presente artículo es una necesidad a nivel mundial, tanto a nivel colectivo como individual.

Si bien en muchos países se han presentado proyectos de educación financiera, estos son o han sido temporales. Cabe resaltar que este tipo de proyectos por lo general lo realizan en cooperación los órganos gubernamentales con entidades financieras privadas, lo cual representa que no existe un profundo interés en la educación financiera más allá de realizar capacitaciones temporales.

El grado de formación en el que las personas deberían llevar la educación financiera como materia curricular es en el nivel secundario, que dependiendo el país varia en años de estudio, por lo cual cada país debería realizar un análisis académico en cuanto a este tipo de formación y adecuarlo a su sistema educativo. 
A su vez, es preciso mencionar que existe un presupuesto del gobierno de cada país destinado a la educación, sin embargo sería interesante por lo menos la presentación de una propuesta educacional en el ámbito de las finanzas.

Por otro lado, los puntos básicos pero a su vez fundamentales para que los jóvenes puedan inmiscuirse en la educación financiera son el ahorro, la inversión, presupuesto personal, presupuesto familiar, dinero en efectivo, dinero virtual, bancos, cuentas bancarias, tarjetas de débito, tarjetas de crédito, compras por internet, seguridad de los datos personales y estafas piramidales.

Si bien las personas cuentan con cierto grado de conocimiento en el área financiera, gran parte de estas personas han aprendido lo que saben de manera empírica, es decir que no han formado parte de un proceso formativo ni siquiera básico.

Se ha logrado identificar la necesidad de las personas por formar parte de un algún tipo de capacitación en educación financiera, haciendo énfasis en que un proceso de esta índole sería muy beneficioso para su posterior aplicación y que este les ayudaría en gran magnitud a administrar de manera más eficiente sus recursos financieros, sean estos sueldos, salarios, mesadas y otros.

Por último, si la educación financiera se implementase de manera curricular en la formación a nivel secundario, se evitarían diversos problemas tales como las estafas, falta de conocimiento en el área financiera, se evitarían a su vez la mala administración del dinero por parte de cualquier persona, lo cual convierte este apartado realmente en una necesidad de la sociedad para que esta se complemente de acuerdo al avance de la tecnología y de muchos otros factores que influyen en la correcta administración de recursos financieros tanto personales como familiares, a su vez, a los jóvenes se les presentaría un adecuado panorama del área y temas inherentes al mismo para que estos de manera más eficiente puedan aplicar dichos conceptos.

\section{Referencias bibliográficas}

Asobancaria (2016). Programas de educación financiera en América Latina: Un énfasis en el caso colombiano. Vol. 1070. 
BAC CREDOMATIC (2008). Libro maestro de educación financiera, un sistema para vivir mejor. Innova Technology: Costa Rica. Vol.1.

Banco de España, Comisión Nacional del Mercado de Valores - CNMV y Ministerio de Educación (2010). Educación financiera en enseñanza secundaria obligatoria. Guía para el alumnado. S/E: España.

Banco de España, Comisión Nacional del Mercado de Valores - CNMV y Ministerio de Educación (2010). Educación financiera en enseñanza secundaria obligatoria. Guía para el profesorado S/E: España.

Corporación Andina de Fomento - CAF (2013). La educación financiera en América Latina y el Caribe, situación actual y perspectivas. Cyngular: Caracas, Venezuela, 15p.

Corporación Andina de Fomento-CAF y Superintendencia de Bancos e Instituciones Financieras de Chile-SBIF. (2016). Encuesta de medición de capacidades financieras en los países andinos: Chile 2016. SBIF: Chile.

Standard \& Poors Ratings (S/A). FinancialLiteracyAroundTheWorld: insightsfromthe standard \&poor's ratings services global financialliteracysurvey.En:http://gflec.org/wp-content/uploads/2015/11/ Finlit_paper_16_F2_singles.pdf

OECD/INFE (2013), Financialeducationforyouth and in schools: OECD/INFE policyguidance,challenges and case studies, in OECD/INFE Set of Criteria, Principles, Guidelines and PolicyGuidancetoImproveFinancialEducation, Part 2: AddressingYouths' and Women'sNeedsforFinancialEducation, available En: finlitedu.org/team-downloads/evaluation/oecdinfe-setofcriteria-principles-guidelines-and-policy-guidance-to-improve-financialeducation-part-2-addressing-youths-and-womens-needs-for-financ.pdf 